\title{
Transparência de Software Centrada na Análise de Sites
}

\author{
Luiz Paulo Carvalho ${ }^{2}$, Claudia Cappelli ${ }^{1,2}$, Flávia Maria Santoro ${ }^{1}$ \\ ${ }^{1}$ DIA-Departamento de Informática Aplicada \\ ${ }^{2}$ PPGI- Programa de Pós-graduação em Informática \\ Universidade Federal do Estado do Rio de Janeiro (UNIRIO) \\ Av. Pasteur, 296 - Urca - CEP 22290-240 - Rio de Janeiro - RJ - Brasil \\ \{luiz.paulo.silva, claudia.cappelli, flavia.santoro\}@uniriotec.br
}

\begin{abstract}
The main goal of certain government sites is to present data, processes, and rules, considering the need for compliance with the Law of Access to Information. Nevertheless, not all of them fulfill basic requirements for transparency, often exposing the required elements without due structures of access, use, quality, understanding, and audibility. This paper proposes a questionnaire that allows evaluating if a site meets the characteristics of transparency through the operations defined in the Catalog of Software Transparency.
\end{abstract}

Resumo. Determinados sites possuem como função principal apresentar dados, processos e regras, atendendo às iniciativas públicas para conformidade da organização com a Lei de Acesso à Informação. Apesar disso, nem todos cumprem requisitos básicos de transparência, expondo muitas vezes os elementos requeridos sem as estruturas devidas de acesso, uso, qualidade, entendimento e auditabilidade. Este trabalho propõe uma avaliação de sites através de um questionário que permita avaliar se estes atendem às características da transparência através das operacionalizações definidas no Catálogo de Transparência de Software.

\section{Introdução}

$\mathrm{O}$ acesso às informações referentes às instituições públicas é garantido ao cidadão pela Lei de Acesso à Informação (LAI) ou Lei 12.527 (Brasil, 2011), regulamentada pelo decreto $\mathrm{N}^{\mathrm{0}} 7.724$ (Brasil, 2012) criando mecanismos que possibilitam, a qualquer pessoa, física ou jurídica, sem necessidade de apresentar um motivo explícito de uso da informação, o recebimento de informações públicas dos órgãos e entidades. Tais informações podem ser apresentadas de forma ativa, por requerimento do cidadão, ou passiva, disponibilizadas espontaneamente e voluntariamente pela organização.

A Corregedoria Geral da União (CGU), a partir do decreto regulamentado, ordenou a operacionalização de iniciativas de transparência nos alvos previstos em lei, entre eles as universidades federais de ensino superior. Estas instituições possuem iniciativas e políticas próprias de transparência para garantir a execução da LAI e assim estar em conformidade com a fiscalização e monitoramento da CGU. Analisando alguns exemplos como a Universidade Federal Fluminense (UFF) ${ }^{1}$ e Universidade Federal do

\footnotetext{
${ }^{1} \mathrm{https}: / /$ sistemas.uff.br/transparencia
} 
Estado do Rio de Janeiro (UNIRIO) ${ }^{2}$, podemos ver que estas possuem sites centralizados específicos para este fim. Neste trabalho serão apresentadas as características da UNIRIO.

Para suprir esta demanda, a UNIRIO criou a Comissão Permanente de Transparência Organizacional (CPTO) que tem como objetivo executar e implantar a LAI na instituição. Este trabalho é regulado pela Ordem de Serviço 004, de 25 de maio de $2012^{3}$. Dois grupos de trabalho foram iniciados por Portarias determinadas, em 2012 e $2015^{4}$. A iniciativa foi delegada desde seu início à Pró Reitoria de Planejamento (PROPLAN), sendo responsável pela constituição e encarregada da CPTO atualmente (Portaria n. ${ }^{0}$ 925, de 10 de outubro de 2016) ${ }^{5}$. A PROPLAN esteve à frente pois a ela é subordinada a Diretoria de Avaliação e Informações Institucionais (DAINF) ${ }^{6}$.

Todas as iniciativas usaram a Internet como meio para transparência ativa e passiva (Fung et al. 2007), utilizando sites para este fim. Inicialmente, as informações eram disponibilizadas em uma página específica, vinculada à página principal da instituição. Posteriormente, passaram para um site específico, centralizando todas as informações da organização que, como ordena lei, devem ser transparecidas. São disponibilizados também formulários para transparência ativa, onde o cidadão pode, sem motivo explícito, requerer informações (aquelas que a lei garante acesso).

Dentre os meios mais acessíveis e disponíveis de apresentar informação pública, um deles é a Tecnologia da Informação e Comunicação (TIC) (Davies, 2015) como a Internet, possibilitando mais alcance e acessibilidade. Na UNIRIO, todas as estruturas que participam da arquitetura organizacional devem apresentar suas informações como ordena a LAI, sendo uma missão não apenas centralizada, mas também fragmentada em cada Unidade Administrativa, Centro, Escola e Programa. Centralizando as informações ortogonais a todas unidades encontra-se um portal de transparência como indexador das diversas estruturas.

Um dos desafios apresentados pela CPTO é padronizar e criar uma identidade visual para os sites da organização, de forma que eles atendam aos requisitos e boas práticas do Web Design, da arquitetura da informação e da LAI. Entretanto, surge um desafio transversal a este: os três itens citados operacionalizados corretamente objetivam conformidade mandatória a transparência, não cobrindo seus aspectos nãofuncionais. A disponibilidade dos dados não garante que, de fato, haja qualidade na transparência, e.g. o website pode não ser interpretado pela maioria dos navegadores; as informações podem estar em extensões impróprias de leitura e uso (como tabelas em formato de imagem); os scripts e plug-ins podem não estar acessíveis; objetos de impossível rastreamento; páginas desconexas do resto do mapa do site; informações confusas e termos complexos; informações sem fontes confiáveis; etc. são exemplos em conformidade com a LAI, mas que efetivamente limitam a transparência. A transparência como objetivo, aplicada, deve também estar alinhada com seus atributos não-funcionais, visando não apenas sua mera operacionalização como sua qualidade efetiva, que garanta ao cidadão, de fato, a transparência (Cappelli, 2009).

\footnotetext{
${ }^{2} \mathrm{http}: / / \mathrm{www} 2 . u n i r i o . b r /$ unirio/lai

${ }^{3} \mathrm{http}: / /$ www.unirio.br/proplan/ordem-de-servico-004-25-05-12-lai

${ }^{4} \mathrm{http}: / /$ www.unirio.br/proplan/transparencia-unirio

${ }^{5} \mathrm{http}: / /$ www.unirio.br/proplan/Portaria109209.12.2016NomeiaCPTO.pdf

${ }^{6} \mathrm{http}: / /$ www.unirio.br/proplan/dainf
} 
Os conceitos de transparência de software (Leite e Cappelli, 2008) podem ser utilizados neste domínio específico, tendo em vista a ampla disponibilidade de informações, a tendência à personalização das aplicações de acordo com o perfil dos usuários e da complexidade dos softwares e sites. Este viés constrói a proposta que visa solucionar o problema exposto neste trabalho, experimentado pela CPTO e também pelos demais websites das iniciativas de transparência: "Como mensurar e garantir, efetivamente e pelas boas práticas, a transparência em sites, objetivando ou não este fim?". E sendo o objetivo final deles transparecer dados, processos ou regras: "O site realmente habilita a transparência?".

A proposta deste trabalho é a criação de um questionário online com perguntas sobre transparência de sites, de acordo com o Softgoal Interdependency Graph (SIG) de Transparência, apresentado por Cappelli (2009), expondo exemplos observáveis, glossário para usuários leigos, resposta em escala para possível sistema de mensuração da transparência, interface simples e intuitiva, possibilidade de feedback sobre a iniciativa, etc. Este artefato deverá auxiliar a CPTO da UNIRIO na construção/revisão de seus websites (Institucional, Escolas, Centros, etc.) deixando-os em conformidade com o SIG de Transparência.

O artigo está estruturado da seguinte forma: Seção 2 apresenta o conceito de transparência de software, que é a base para o assunto da Seção 3, transparência de sites, a Seção 4 expõe a proposta de formulário online para aplicar e mensurar a transparência de sites, a Seção 5 conclui o trabalho.

\section{Transparência de Software}

Transparência pode ser obtida através da implementação de um conjunto de características (Cappelli, 2009). Cappelli (2009) utilizou um SIG (Chung et al. 2000) para representar o conceito de transparência (Figura 1). Este conceito foi aplicado para o domínio da Engenharia de Software, tendo como tópico o software (Leite, 2010).

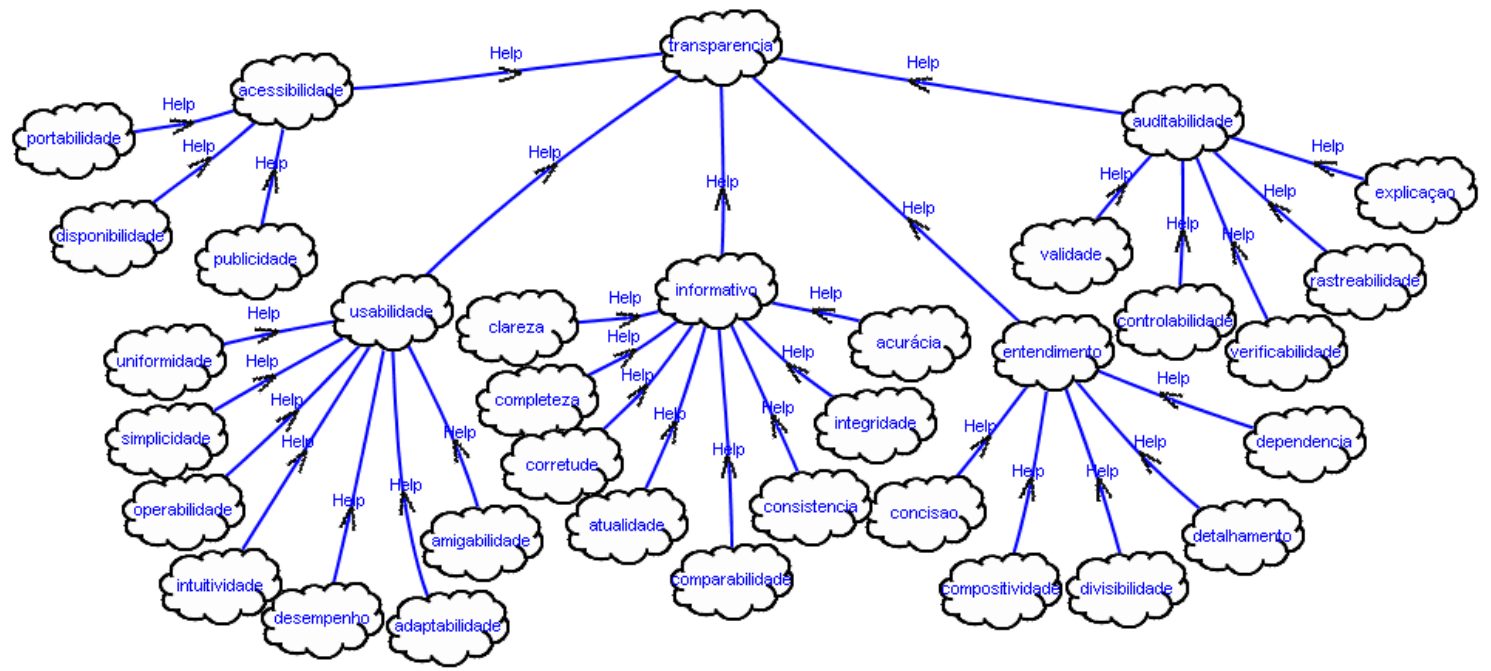

Figura 1: SIG de transparência organizacional, proposto por Cappelli (2009).

Cada característica tem definições como demonstradas na Tabela 1. 
Tabela 1 - Descrição das Características do SIG de Transparência. Cappelli (2009).

\begin{tabular}{|c|c|c|}
\hline \multicolumn{2}{|c|}{ Características } & $\begin{array}{c}\text { Descrição. } \\
\text { Tem capacidade de... }\end{array}$ \\
\hline \multirow{3}{*}{ Acessibilidade } & Portabilidade & Ser utilizado em diversos ambientes. \\
\hline & Disponibilidade & Aceitar solicitações. \\
\hline & Publicidade & Ser conhecido. \\
\hline \multirow{6}{*}{ Usabilidade } & Uniformidade & Manter uma única forma. \\
\hline & Intuitividade & Ser utilizado sem aprendizado prévio. \\
\hline & Simplicidade & Não apresentar dificuldades ou obstáculos. \\
\hline & Amigabilidade & Uso fácil. \\
\hline & Operabilidade & Ser executado. \\
\hline & Desempenho & Operar adequadamente. \\
\hline \multirow{8}{*}{ Informativo } & Clareza & Prover informações com nitidez. \\
\hline & Acurácia & Ausência de erros. \\
\hline & Completeza & Não faltar nada do que pode ou deve ter. \\
\hline & Corretude & Ser isento de erros. \\
\hline & Consistência & $\begin{array}{l}\text { Resultar aproximadamente em várias medições } \\
\text { de um mesmo item. }\end{array}$ \\
\hline & Integridade & Ser preciso e rigoroso. \\
\hline & Comparabilidade & $\begin{array}{l}\text { Ser confrontado com outro para lhe determinar } \\
\text { diferença, semelhança ou relação. }\end{array}$ \\
\hline & Atualidade & Refletir a última informação ou mudança. \\
\hline \multirow{4}{*}{ Entendimento } & Compositividade & $\begin{array}{l}\text { Construir ou formar a partir de diferentes } \\
\text { partes. }\end{array}$ \\
\hline & Concisão & Ser resumido. \\
\hline & Divisibilidade & Ser particionado. \\
\hline & Adaptabilidade & $\begin{array}{l}\text { Mudar de acordo com as circunstâncias e } \\
\text { necessidades. }\end{array}$ \\
\hline \multirow{5}{*}{ Auditabilidade } & Explicável & Explicar os dados, procedimentos e regras. \\
\hline & Rastreabilidade & $\begin{array}{l}\text { Seguir o desenvolvimento de um processo ou a } \\
\text { construção de uma informação, suas mudanças } \\
\text { e justificativas. }\end{array}$ \\
\hline & Verificabilidade & $\begin{array}{l}\text { Ser legitimado, identificar se o que está sendo } \\
\text { feito é o que deve ser feito. }\end{array}$ \\
\hline & Validade & $\begin{array}{l}\text { Ser testado por experimentação ou observação, } \\
\text { identificar se o que está sendo feito é correto. }\end{array}$ \\
\hline & Controlabilidade & Ser controlado. \\
\hline
\end{tabular}

Cada uma das características é implementada a partir de uma ou mais operacionalizações. Elas têm como objetivo analisar e avaliar a prática das características, e.g. a concisão pode ser alcançada fazendo uso de resumos ou opções de expansão para visualizar "mais detalhes"; uniformidade é operacionalizada com 
consistência entre informações, páginas, layout, etc.; adaptabilidade pode ser alcançada quando se permite ao usuário que utilize dados diferentes com o mesmo valor semântico (se não tiver $\mathrm{CPF}$, então o passaporte para identificá-lo); uma diagramação bem arquitetada e implementada gera mais simplicidade, uma outra operacionalização da simplicidade é o uso consciente e com boa prática das cores; etc.

Para um software ser dito totalmente transparente ele deve estar habilitado com todas as características descritas na Tabela 1 através de operacionalizações específicas para software. Um software é considerado transparente quando: (i) apresenta informações de forma transparente (transparência de informações geradas pelo software) e (ii) quando apresenta informações sobre si, como opera, o que faz e o motivo (transparência do processo de desenvolvimento de software) (Leite e Cappelli, 2008). O Catálogo de Transparência de Software ${ }^{7}$ apresenta características e operacionalizações específicas para atender a estes dois tipos de transparência de softwares em geral.

Como exemplos de transparência de software temos: o uso e manutenção dos padrões de nomes (como variáveis, funções, módulos, etc.), podendo ser operacionalizada também na Web com os seletores de id e classe no Cascade Style Sheet (CSS) (uniformidade); resolução e aprimoramento do tempo de falha (operabilidade); organização do tamanho dos módulos e das sentenças no código (intuitividade); disponibilização do código de forma aberta (adaptabilidade); entre outras. Estes tipos de operacionalizações de transparência de software se enquadram na transparência do processo de desenvolvimento de software, não se relacionando diretamente à LAI.

\section{Avaliação de Transparência em sites}

Para avaliação da transparência em sites de diferentes domínios (ex. sites de compra e venda, sites de empresas telefônicas e sites públicos) foi proposto um questionário desenvolvido durante a construção do Catálogo de Transparência (Cappelli, 2009). Este questionário ${ }^{8}$ é composto de 93 perguntas que permitem investigar as operacionalizações de cada característica definida (Cappelli, 2009). Cada pergunta possui um objetivo, uma categorização de respostas, utilizando a Escala Likert (Likert, 1932) e justificativas.

O questionário é dividido em seções que representam as características da Tabela 1 de acordo com o SIG de Transparência (Cappelli, 2009). As subseções apresentam as demais características, formando um modelo detalhado para análise de transparência em sites. $O$ uso de perguntas tem o propósito de investigar $o$ comportamento do site quanto a transparência usando como base a própria opinião dos usuários.

O modelo para a apresentação das perguntas é descrito na Figura 2. O mesmo é composto de três áreas principais: i) área reservada para a pergunta e exemplos; ii) área reservada para os objetivos e as justificativas; e iii) área reservada para questionamentos, esclarecimentos extras, comentários e, caso necessário, glossário. Foram usadas cores, espaçamentos e um layout em tabela para facilitar o entendimento

\footnotetext{
${ }^{7}$ http://transparencia.inf.puc-rio.br/wiki/index.php/Catálogo_Transparência

${ }^{8} \mathrm{http}: / /$ pes.inf.puc-rio.br/questionario/index.html
} 
por parte dos usuários. Cada característica agrupadora foi definida como um critério de avaliação e as demais foram definidas como subcritérios. Cada uma delas foi explicada usando uma descrição acerca do seu significado. As descrições foram adaptadas a partir das descrições da Tabela 1 para atenderem ao tópico de sites. Essas descrições ficam disponíveis para consulta dos usuários. A Figura 2 apresenta a descrição para "Acessibilidade" / "Portabilidade".

1 - Critério Acessibilidade: Capacidade de acesso.

1.1. Sub-critério Portabilidade: Capacidade de ser interpretado em diferentes navegadores.

Questão 1: O site pode ser acessado por diferentes browsers? Ex.: O site das lojas Americanas pode ser acessado via Mozilla Firefox, Google Chrome, dentre outros.

* Objetivo: Investigar se o site está preparado para ser acessado usando qualquer browser, seja através de plug-ins ou outras tecnologias apropriadas.

* Justificativa: Garantir a portabilidade em diferentes browsers para facilitar o acesso aos conteúdos oferecidos pelo site.

( ) POR TODOS OS BROWSERS QUE CONHEÇO

( ) PELA MAIORIA DOS BROWSERS QUE CONHEÇO

( ) PELA MINORIA DOS BROWSERS QUE CONHEÇO

( ) POR APENAS UMI BROWSER QUE CONHEÇO

( ) POR NENHUM DOS BROWSERS QUE CONHEÇO

( ) NDA

Se possivel, especifique quais browsers tentou e quais funcionaram:

Comentários:

Figura 2: Exemplo de pergunta do questionário

Esta proposta alinha-se ao paradigma de transparência proposto por Cappelli (2009), diferenciando-se de demais abordagens com características semelhantes dedicados ao domínio Web. Acessibilidade, por exemplo, no contexto de Web significa que pessoas com deficiências possam usá-la (Henry e Arch, 2012), como cegos, idosos, pessoas com baixo nível de instrução, utilizando equipamentos antigos, etc.; usabilidade assegura que algo funcione bem, uma pessoa com habilidade e experiência comuns possa usar a Web para seu propósito desejado sem ficar frustrada com isso (Krug, 2014) (Nielsen, 1993), como diagramação, área de clique, aparência, abas, migalhas, etc. A acessibilidade e usabilidade do contexto de transparência diferem da Web, já que possuem escopos diferentes. Não necessariamente um site transparente (que corresponda a todas as características) será um site com boa qualidade pelos critérios da Web, e vice-versa.

\section{Proposta de formulário para avaliação de sites}

A abordagem citada na seção anterior encontra-se disponível online ${ }^{8}$. Porém, como foi desenvolvida durante a construção do SIG de Transparência, a última versão e suas operacionalizações estão defasadas frente ao SIG de Transparência atual pois algumas de suas características foram alteradas, e.g., a característica de Divulgação se tornou 
Publicidade na última versão do SIG de Transparência. Para o questionário atualizado e aprimorado deverá ser criado um novo formulário online. Todos os elementos e decisões de conteúdo existentes na versão atual serão mantidos, sendo eles:

* Uso de alternativas: como se trata de um questionário extenso, optou-se pelo uso de alternativas para estimular a participação dos voluntários e simplificar as respostas.

* Uso de cores nas alternativas: serão usadas cores em tons graduais. A analogia escolhida como base para o uso das cores está na associação com notas vermelhas e azuis e no uso de uma cor básica (cinza) para representar a neutralidade. Quanto mais próximo de um comportamento transparente o site estiver, mais azul é a cor da alternativa, utilizando o mecanismo da Escala Likert (Likert, 1932). Quanto mais desaprovado o comportamento do site em termos de transparência, mais vermelha é a cor da alternativa. O usuário pode ainda poderá optar pela neutralidade, quando o site é "parcialmente" transparente.

* Uso de barras para divulgação dos resultados parciais aos usuários: optouse por usar barras indicando os resultados parciais da pesquisa. Esse resultado será apresentado ao usuário sempre que o mesmo responder um conjunto de perguntas relacionadas a um determinado subcritério. Não é possível a visualização prévia (antes de responder as perguntas), para não influenciar as respostas.

* Uso de objetivos e justificativas para cada pergunta: o uso de objetivos e justificativas, além de ajudar no refinamento do questionário, também visará melhorar o entendimento e reforçar o propósito de cada pergunta.

* Uso de ocultação dos detalhes: alguns detalhes serão ocultados usando o símbolo $(+)$ para não "poluir" demais a interface com informações que nem sempre agradam os usuários e melhorar a concisão. Entretanto, será permitida a visualização dessas mesmas informações por aqueles que desejam obter maiores detalhes.

* Uso de tabelas divididas em áreas específicas para cada informação disponibilizada no formulário: será usada apresentação gráfica em tabela, dividida em áreas para a postagem específica de informações. Essa prática padroniza a exposição de ideias, deixando as informações mais claras.

* Uso de seções e subseções para cada critério e seus subcritérios: serão usadas seções e subseções visando: i) facilitar a exposição das informações; ii) facilitar o entendimento do usuário; e iii) avaliar separadamente cada critério de transparência e seus subcritérios.

* Uso de exemplos na maior parte das perguntas: serão adotados exemplos, reais, genéricos ou fictícios, para a maioria das perguntas com o objetivo principal de esclarecer possíveis dúvidas dos usuários quanto ao propósito de cada pergunta.

A usabilidade, eficácia e eficiência da abordagem desatualizada foram avaliadas como favoráveis ao objetivo da mesma (Cappelli, 2009). Logo, seus elementos serão mantidos, sendo feitas as devidas atualizações de forma a estar compatível com a última versão do SIG de Transparência e também incluindo algumas melhorias quanto a clareza e comparabilidade de informações:

* Responsividade: a nova interface será responsiva, para melhor uso em aparelhos móveis e tablets; 
Atualização das perguntas e respostas: melhorar a clareza e objetividade das respostas, resolver problemas semânticos e esclarecer itens duvidosos;

* Revisão dos exemplos das perguntas: revisar e atualizar os exemplos presentes nas perguntas, tendo em vista que os sites dos exemplos são atualizados. Buscar novos exemplos para as perguntas ainda sem exemplos;

* Glossário: perguntas com termos técnicos complexos, e.g. plug-in, terão um glossário simples ou condução para outro site explicativo para que o questionário tenha maior alcance, não sendo apenas utilizado por especialistas do domínio Web;

* Mecanismo de valoração, quantificação e comparação de transparência: permitir comparação entre sites no aspecto transparência, permitindo que eles não sejam apenas avaliados como comparados e valorados dependendo das respostas no formulário. Duas opções são propostas: avaliar a transparência individualmente, categorizando em uma escala de transparência, e comparativamente entre dois ou mais sites, notificando qual possui melhor transparência e qual característica um tem melhor que o outro. As perguntas e respostas precisam ser atualizadas para este fim;

* Revisão com base em tecnologias Web atualizadas: Adequar o artefato a novas tecnologias, como HTML 5 e CSS 3, não disponíveis na época em que foi construído.

A Figura 3 expõe um exemplo de uma pergunta incluída na nova versão do questionário, da característica "Informativo" / "Acurácia" utilizando as decisões descritas.

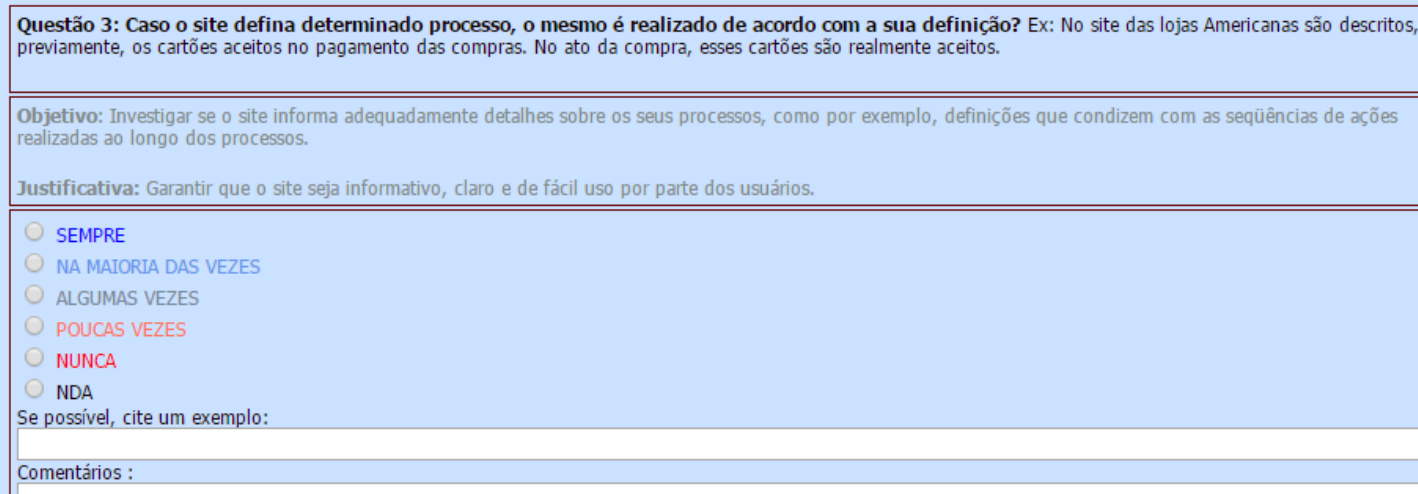

Figura 3: Proposta de como será exposta uma pergunta do questionário no formulário online.

\section{Conclusão}

Este trabalho buscou apresentar como avaliar a transparência centrada na análise de sites. Seu principal objetivo foi buscar mecanismos que possam auxiliar a CPTO da UNIRIO a habilitar a transparência em seus sites informacionais. Esse trabalho usou como base uma primeira iniciativa de análise de transparência em sites apresentada na Seção 3, onde foram determinadas as principais características que ajudam a analisar transparência. Estas características foram usadas como critérios, de acordo com o SIG de Transparência. Os critérios contemplados na análise são: acessibilidade, usabilidade, informativo, entendimento e auditabilidade. Associados a esses critérios existem vários 
outros subcritérios (ex. portabilidade, clareza e validação) que também foram utilizados visando uma análise mais refinada e modularizada. Como instrumento de avaliação de sites sob a perspectiva da transparência, foi desenvolvido um questionário.

Atualmente, este questionário encontra-se desatualizado pois foi construído ao longo do desenvolvimento do SIG de Transparência. A proposta deste trabalho foi revisar este questionário já existente, verificando o que deve ser mantido e definindo como este deve ser atualizado, incluindo também alguns novos elementos para melhoria do mesmo.

Como trabalho futuro será implementada sua versão online, o Formulário Transparência Online. Será também implementado um banco de dados e elaborados os desenhos lógico e físico do mesmo. Buscar-se-ão voluntários para utilização do formulário, armazenadas e analisadas suas respostas para averiguar a utilização do artefato. Realização de estudos para que o artefato abranja também plataforma mobile.

Um dos desafios observados é que como a TIC, incluída nela a Web, está em constante desenvolvimento e mudança, desta forma, o formulário online deve ser atualizado constantemente, verificando se os exemplos ainda são reais e usáveis, se não há necessidade de mais perguntas, mudanças nas perguntas atuais, etc.

\section{Referências}

Brasil, DECRETO No 7.724, DE 16 DE MAIO DE 2012. Disponível em: http://www.planalto.gov.br/ccivil_03/_ato2011-2014/2012/decreto/d7724.htm. Acesso em: 03/03/2017.

Brasil, LEI $\mathrm{N}^{\mathrm{o}}$ 12.527, DE 18 DE NOVEMBRO DE 2011. "Lei de Acesso à Informação". Disponível em: http://www.planalto.gov.br/ccivil_03/_ato20112014/2011/lei/112527.htm. Acesso em: 03/03/2017.

Cappelli, C. (2009) "Uma Abordagem Para Transparência Em Processos Organizacionais Utilizando Aspectos". Tese de Doutorado, Pontifícia Universidade Católica do Rio de Janeiro, Brasil.

Chung, L., Nixon, B. A., Yu, E., Mylopoulos, J. (2000) "Non-Functional Requirements in Software Engineering." Kluwer Academic Publishers, Londres.

Davies, R. (2015) "eGovernment: Using technology to improve public services and democratic participation". Serviço de Pesquisa do Parlamento Europeu, União Europeia.

Fung, A., Graham, M. e Weil, D. (2007) "Full Disclosure: The Perils and Promise of Transparency". Cambridge University Press, Estados Unidos da América.

Henry, S., Arch, A. (2012) "Developing a Web Accessibility Business Case for Your Organization". W3C. Disponível em: https://www.w3.org/WAI/bcase/ Acesso em: 03/03/2017.

Krug, S. (2014) "Don't Make Me Think, Revisited: A Common Sense Approach to Web Usability". New Riders Publishing, Estados Unidos da América.

Leite, J. C. S. P. (2010) "Software Transparency". Procedimentos do $13^{\circ}$ Congresso Iberoamericano em Engenharia de Software. 
Leite, J. C. S. P., Cappelli, C. (2008) "Exploring i* Characteristics that Support Software Transparency". Procedimentos do $3^{\circ} \mathrm{i}^{*}$ Workshop Internacional.

Likert, R. (1932) "A Technique for The Measurement of Attitudes". Universidade de Nova Iorque, Arquivos de Psicologia.

Nielsen, J. (1993) "Usability Engineering". Morgan Kaufmann, Estados Unidos da América. 